\title{
Research, ethics and conflicts of interest
}

\author{
Miles Little University of Sydney, Australia
}

\begin{abstract}
In this paper, I have tried to develop a critique of committee procedures and conflict of interest within research advisory committees and ethical review committees (ERCs). There are specific features of conflict of interest in medical research. Scientists, communities and the subjects of research all have legitimate stakeholdings. The interests of medical scientists are particularly complex, since they are justified by the moral and physical welfare of their research subjects, while the reputations and incomes of scientists depend on the success of their science. Tensions of this kind must at times produce conflict of interest. It is important to recognise that conflicts of interest may unwittingly lead to manipulation of research subjects and their lay representatives on research committees. It is equally important to recognise distinctions between the legal and moral aspects of conflict of interest. Some practical suggestions are made which may go some way towards resolving these difficulties. They indicate what might be needed to ensure the validity of ethical discourse, and to reduce the risks associated with conflict of interest.
\end{abstract}

(Fournal of Medical Ethics 1999;25:259-262)

Keywords: Conflict of interest; research advisory committees; stakeholdings; discourse

Health research is an enterprise which is morally grounded and morally justified. Research advisory committees have the responsibility to sponsor good science, because it would be immoral to fund bad science. But there is also a moral responsibility to relate the science to the real needs of those who suffer from the diseases under study. Further, there is a moral responsibility to remain alert to possible conflicts of interest, since they can interfere in many ways with the justice and equity of the research funding process. Science, the community and the subjects and possible beneficiaries of research all have legitimate stakeholdings in health research. This threeway relationship is complicated by the internal complexities of each of these groups.

It is also now imperative for research proposals to undergo ethical review, frequently by more than one ethical review committee. Ethical review adds another layer of complexity. Since expert members of ethical review committees may also have vested interests in seeing ethical problems resolved in particular ways, further possible conflicts of interest may be created.

\section{A theory of research and ethical review}

McNeill has evolved a theory of committee representation which might deal with conflicts of interest on ethical review committees. ${ }^{1} \mathrm{He}$ concludes that the stakeholders in research must be correctly represented. Thus, the ethical review committee must represent expertise (science and scientists), the laity (community representatives), and subjects (consumer groups). Members of committees will assume different roles at different times. "Lay" members are likely to have expertise in matters such as the law, theology or ethics, and subject representatives may be chosen for their familiarity with the principles of scientific research. All committee members should see themselves as representing the subjects of research at all times, whatever their reason for appointment. It is clear, therefore, that there need to be procedural rules which would govern the way in which these representations were validated and guaranteed their due influence. These rules are those which govern constructive discourse.

Habermas has suggested that discourse is the only mode in which moral understanding can be constructed. ${ }^{2}$ In order to achieve this end, he proposes strict rules which should govern ethical discourse, rules derived from the work of Alexy. ${ }^{3}$ These are stringent, and it is worthwhile to set them out in some detail. They specify that practical discourse should exist at certain levels, and that particular rules are appropriate for each level. I stress that what follows is a "translation" of the material set out in a formal way by Alexy. Those interested in this formal statement will find it in an appendix at the end of this paper.

The first level is that of logic and meaning. No speaker is allowed to contradict her or him -self (obvious but not always followed). Speakers must be consistent in their valuations of topics under discussion. Everyone has to agree on the meanings of the terms used in the discussion. After the meeting has agreed on what is meant by a term 
such as "informed consent", no one can change that meaning and develop arguments based on another meaning of the term.

At the second, procedural level, speakers must commit and discipline themselves to say only what they truly believe. Claims made for the sake of securing an advantage in a bargaining process have no place in constructive discourse. Further, any person who wants to change the grounds of the discussion by challenging basic values and norms that are not under discussion, must give good reasons for doing so. Having agreed that controlled trials are desirable because they produce the "best" scientific evidence, it cannot then be argued that a drug should be made freely available because there are unsubstantiated rumours that it may be effective.

At the third level of process, every legitimate stakeholder in the subject of the discourse has a right to speak and be heard. Every participant can question the validity of any assertion, regardless of who has made that assertion. In turn, it has to be accepted that anyone can introduce any assertion, provided the assertion conforms to the rules of relevance and sincerity. Thus, all can and should express their desires, values and needs so that others can understand the basis and direction of their arguments. Finally, those people who qualify as legitimate stakeholders cannot be prevented, either by other parties to the discourse or by people or groups outside the discourse, from saying what they sincerely believe.

In their formal way, these rules suggest that commitment to reality, and honesty to self, group and cause are fundamental to constructive discourse. What Alexy says closely parallels the conclusions of Arrow and his colleagues in their work on conflict resolution. ${ }^{4}$ It is thus possible to find procedural rules that might govern the type of discourse which ethical review committees might undertake. Within these rules, conflict of interest would be managed by open declaration of conflict, and by a process of distancing and dissociation of those with an interest from further involvement in those procedures which involve the conflict.

This discussion suggests that there should be a clear separation of powers and functions between committees that allocate funds for research, and those that undertake ethical review of research. An ethical review committee might wish to suggest or be asked to suggest ways in which distributive justice can best be ensured. For one committee to fulfil both distributive and ethical review functions, however, would involve a de facto conflict of interest, since ethical review may well

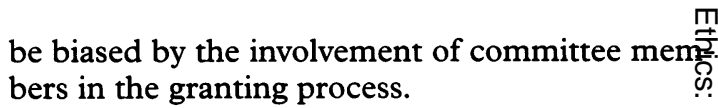

It is clear that the members of both research advisory committees and ethical review commiff tees, like the board members of public companies, undertake serious responsibilities. They need be briefed on these responsibilities, and trained carry them out. The frank declaration of confli go of interest is a part of the modern committe member's obligation to herself, her institution, he्P committee and her community. Once the interest has been declared, committees and their chairs age obliged to decide what part can be played 啇 relevant deliberations by the member with corp flicting interests.

\section{Power}

The question of the power imbalance in medicine has been addressed by Foucault ${ }^{5}$ and Brody ${ }^{6}$ among many others. Power imbalance is morals undesirable in medical research because it makes manipulation of others easily possible. It gives particular meaning to the threat of conflict of interest. People can be turned from ends to mere means by the persuasions of those with vested interests who have implicit or explicit powers dominate discussion. Medical scientists, like other scientists, have command of specialis knowledge. When they communicate this know edge to one another, they employ a language which is convenient to them, and which expresses their collegiality. This specialised vocabulary and usage are justified because they are employed communicate specific knowledge in the shortest way. Words used in these ways, however, maty carry large portmanteaux of meaning and implicition, which may be completely lost on those whi do not know the scientific jargon. Further, non-cognate members of research and ethicăl review committees may not enjoy the sanye relevant collegiality. Representatives of churches, government agencies and other academic disc? plines will have collegial support beyond the conff mittee, but not necessarily in the area of thes science under discussion. The cognate member ship, therefore, can readily draw on one another for support in discussions and disagreements, bफ़् non-cognate members have no such support at the time of the discussion. They can too easily be out-argued.

Those who represent the subjects of researen have other distinctive vulnerabilities and power. The subjects they represent may be alrea disempowered by illness and by their need 8 endorse research in the area of that illness. On the other hand, research subjects build their power generating their own collegiality and by exerting 
moral force which comes from their existence as humans with problems which justify research. They gather strength from the need of the researchers to have the approval of their subjects in order to defend and validate their research, and from their own capacity to identify issues for further research. In some areas, such as AIDS, their lobbying power may be so formidable that there is a real chance that they may influence decisions against the advice and judgment of scientists.

The problem remains, however, of developing and maintaining a balance between expertise and representation as these balances of power shift and evolve. In part, this issue can be resolved by accepting that common language is the key to an honesty of communication, which alone can validate research. The plain-language translation of research protocols thus assumes central importance. The ability of the research worker to set out in common language the justification, process and possible ends of the research programme becomes the major determinant of its relevance. This means that those sections of a grant application which require an explanation of the programme and its implications in lay terms become the core of the application. It should no longer be enough to re-state the protocol with less technical language, and then dismiss the assessment of impact on those who suffer from the illness with some such expression as "These results will clearly have major implications for those suffering from this disease". Those implications need to be examined in some detail, and in language which is clearly understood by the noncognate members of committees. Clear language alone can make clear whose interests are to be served and how they might be served.

\section{Ethics and the law}

There is an important distinction to be drawn between the moral and legal content of conflict of interest. The law places considerable importance on disclosure of interest as a means to protect against the potential evils of conflict of interest, particularly when money is involved. ${ }^{7}$ The law may then bring external sanctions to bear if someone fails to disclose an interest. The moral and non-monetary value-content of conflict of interest in professional relationships is at least as important, because conflict of interest undermines the trust upon which the professional-client relationship is constructed. ${ }^{8}$ Indeed, the Canadian Tri-Council's Code of Conduct for Research Involving Humans proposes that "To determine if the researcher is in conflict of interest-whether actual, apparent or potential-the REB [Research Ethics Board] must determine whether the conflict is likely to interfere, or appear to interfere, with the researcher's independent and objective judgement. One test the REB can use is the 'trust test' which asks: would research subjects trust the researcher's scientific judgment if they knew the researcher was in this situation? In the research setting, conflict of interest relates to the trust research subjects have in the researcher and the research enterprise. By undermining this trust, a conflict of interest risks damaging both the reputation of the researcher and the research community". 9

\section{Grounding values}

This paper suggests ways to restore research assessment-both scientific and ethical-to its grounding values. We live in an era of postEnlightenment politics and philosophy. We continue to place a distinctive valuation on individual human life, both for its quantity and quality. We give a particular assignment of meaning to "autonomy", the assumption that each of us is a moral agent with a right and even a necessity to make our own moral choices. Along with that respect for autonomy goes a concern for human suffering. It is these principles of respect for individual human life in its domains of security and self-directing flourishing which justify and enable the moral value of the whole health endeavour. Science has no justification in health in and of itself. It is the value we assign to people's lives that enables the expenditure of so much on health and scientific health research. These values must therefore translate into research theory and practice in the health sciences.

The present climate of resource constraint brings new moral dimensions to the issue. Economics is the management of scarce resources, and it is this economic obligation which sharpens the need for ethical review processes and for proper structure of the reviewing bodies. Such management involves major questions of values and value judgments in making decisions on how best to allocate resources in society's best interests. Additionally, there is an ethical question of whose values and whose value judgments are to count. Our society is determinedly pluralistic. The old moral authorities (the churches, the nationstates) have been perceptibly weakened, and our ethics are progressively constructed by the discourse which we hold with one another as we discuss such issues as those which arise during the deliberations of groups such as research advisory committees and ethical review committees. ${ }^{2}$ Plural and conflicting interests are inevitable, because modern Western societies are constructed in that way. There is thus a clear obligation placed on 
those who take part in these discussions to learn the art of structured discourse (after the patterns proposed by Alexy and others), and to acquire the art of what Arendt call "representative thinking", the practice of engaging sympathetically and honestly with the values and thought processes of people from other walks of life, but with legitimate interests in the issues under discussion. ${ }^{10}$ The ways towards that goal are clearly difficult. To dismiss them, however, as impossible or too hard to follow will be to abrogate a responsibility which research advisory committees and ethical review committees have to the health professions, medical scientists, the subjects of medical research and the community at large.

\section{Appendix}

The formal statement of the Alexy rules is as follows:

I. Logical-semantic level

No speaker may contradict himself.

Every speaker who applies predicate $F$ to object A must be prepared to apply $F$ to all other objects resembling $\mathrm{A}$ in all relevant aspects.

Different speakers may not use the same expression with different meanings.

II. Procedural level

Every speaker may assert only what he really believes.

A person who disputes a proposition or norm not under discussion must provide a reason for doing so.
III. Process level

Every subject with the competence to speak and act is allowed to take part in the discourse.

Everyone is allowed to question any assertiof whatever.

Everyone is allowed to introduce any assertic whatever.

Everyone is allowed to express his attitudes desires and needs.

No speaker may be prevented, by internal $\vec{\theta}$ external coercion, from exercising his rights as laie down in 1 and 2 .

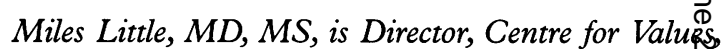
Ethics and the Law in Medicine, Department of Such gery, University of Sydney.

\section{References}

1 McNeill PM.The ethics and politics of human experimentation Cambridge: Cambridge University Press, 1993.

2 Habermas J. Moral consciousness and communicative actif. Cambridge: Polity Press, 1992.

3 Alexy R. A theory of practical discourse. In: Benhabib S, Dalle mayr $\mathrm{F}$, eds. The communicative ethics controversy. Cambridge Mass: MIT Press, 1990: 151-90.

4 Arrow K, Mnookin RH, Ross L, Tversky A, Wilson R, eds.Barriers to conflict resolution. New York: WW Norton and Co, 1905

5 Foucault $M$. The birth of the clinic - an archaeology of medical pe ception. New York: Vintage Books, 1975.

6 Brody H. The healer's power. California: Stanford Univers Press, 1993.

7 Rodwin MA. Medicine, money and morals: physicians' conflicts interest. Oxford: Oxford University Press, 1993.

8 Koehn D. The ground of professional ethics. London: Routledge 1994.

9 Canadian-Tri-Council. Code of conduct for research involvizhg humans. Electronic version, 1997: section 9, article 9.2.

10 Arendt H. Between past and future: eight exercises in politig thought.Harmondsworth: Penguin Books, 1993. 